\title{
Photoluminescence temperature behavior and Monte Carlo simulation of exciton hopping in InGaN multiple quantum wells
}

\author{
K. Kazlauskas ${ }^{{ }^{* 1}}$, G. Tamulatis ${ }^{1}$, P. Pobedinskas ${ }^{1}$, A. Žukauskas ${ }^{1}$, \\ Chi-Feng Huang ${ }^{2}$, Yung-Chen Cheng ${ }^{2}$, Hsiang-Chen Wang ${ }^{2}$, and C. C. Yang \\ ' Institute of Materials Science and Applied Research, Vilnius University, \\ Saulètekio 9, Build. III, 10222 Vilnius, Lithuania \\ ${ }^{2}$ Graduate Institute of Electro-Optical Engineering, National Taiwan University, \\ 1 Roosevelt Road, Sec. 4, Taipei, Taiwan
}

Received 8 July 2004, accepted 19 September 2004

Published online 4 February 2005

PACS 02.70.Uu, 71.23.An, 73.21.Fg, 78.40.Fy, 78.55.Cr, 78.67.De

Application of Monte Carlo simulation of exciton (carrier) hopping for the analysis of the photoluminescence (PL) temperature behavior in $\mathrm{In}_{02} \mathrm{Ga}_{08} \mathrm{~N} / \mathrm{GaN}$ multiple quantum wells is reported. The PL linewidth and peak position measured in the $10-300 \mathrm{~K}$ range exhibited a $\mathrm{W}$-shaped and S-shaped temperature behavior, respectively. The $\mathrm{W}$-shaped linewidth dependence was fitted with the results of Monte Carlo simulation, which involved phonon-assisted exciton hopping through energy states confined in the band potential fluctuation minima. The simulation yielded the values of the standard deviation for potential fluctuations within In-rich regions $(31 \mathrm{meV})$, dispersion of the average exciton energy in different regions $(29 \mathrm{meV})$, and the temperature dependence of the band gap, which was found to be in a fair agreement with the photoreflectance data. Our results, which infer in-plane motion of localized excitons within the wells, are consistent with the model of large In-rich regions ("segmented quantum wells" or "quantum discs") with band potential fluctuations inside these regions.

(C) 2005 WILEY-VCH Verlag GmbH \& Co. KGaA, Weinheim

1 Introduction InGaN/GaN multiple quantum wells (MQWs) are known to efficiently emit green to near UV light in spite of high density of dislocations due to lattice-mismatched substrates [1]. The unique optical properties of InGaN are believed to relate to compositional disorder. In particular, carrier and exciton localization in the band potential fluctuation minima should prevent them from reaching nonradiative recombination sites. However despite a commercial success of nitride-based emitters, the physical origin of efficient light generation in this partially disordered system is unveiled incompletely. In particular, carrier/exciton motion and the establishing of their distribution over the localized states in InGaN are not completely understood. Meanwhile, an anomalous temperature behavior of photoluminescence (PL) in group-III nitride structures designated as an S-shaped peak position dependence and Wshaped linewidth dependence [2-4] indicate that exciton/carrier motion occurs via phonon-assisted tunneling (hopping) through localized states [5,6], what results in incomplete thermalization of localized excitons at low temperature. Recently, an evolvement of the S-shaped and $\mathrm{W}$-shaped temperature dependences of the PL peak position and linewidth, respectively, was observed with gradual incorporation of indium in to AlGaN alloy and accounted for by using Monte Carlo simulation of exciton hopping [7].

Here we show that phonon-assisted hopping can account for nonmonotonous temperature dependences of the PL peak position and linewidth in InGaN MQWs. The PL linewidth temperature behavior is shown to be in quantitative consistence with the energy of the fundamental optical transition deduced from the photoreflectance (PR) measurements [8].

"Corresponding author: e-mail: karolis.kazlauskas@ff.vu.It, Phone: +370 5236 6096, Fax: +370 52366059 
2 Experimental Narrow quantum wells, where the inhomogeneous broadening of the PL band due to fluctuations of the built-in field via the quantum-confined Stark effect (QCSE) is small enough to accurately measure the linewidth, were investigated. The sample grown by metal-organic chemical vapor deposition over a sapphire substrate consisted of five 2.5-nm thick $\operatorname{In}_{x} \mathrm{Ga}_{1-x} \mathrm{~N}(x \approx 0.2)$ quantum wells separated by $9-\mathrm{nm}$ thick GaN barriers deposited on a $2.5-\mu \mathrm{m}$ thick $\mathrm{GaN}$ buffer layer.

A continuous-wave and chopped emission of a He-Cd laser $(325 \mathrm{~nm})$ was used for excitation in PL and PR experiments, respectively. The optical signal was dispersed by a double monochromator and recorded by a photomultiplier. PL was measured in the photon-counting regime. To avoid distortions of the PL line shape due to Fabri-Perrot interference, we collected the PL signal through the frosted transparent substrate. In the PR measurements, the modulated constituent of reflected radiation from halogenlamp was detected by means of a lock-in amplifier. The measurements were performed in the $10-310 \mathrm{~K}$ temperature range.

3 Results and discussion The PL spectra measured at different temperatures are presented in Fig. 1. The spectra contain a single band due to recombination of localized excitons and a longitudinal-opticalphonon replica on the low-energy slope of the principle band. The PL band peak position is highlighted by dots in Fig. 1. The peak exhibits a well-established S-shaped temperature behavior [2-4] (redshiftingblueshifting-redshifting). The inset in Fig. 1 shows an example of a PR spectrum at $295 \mathrm{~K}$ with the indicated band-edge resonance energy deduced by fitting with a Lorentzian lineshape functional form. Note that the PR feature refers to the actual energy of the fundamental optical transition (with the red shift due to the QCSE included). Reliable PR spectra were difficult to obtain below $200 \mathrm{~K}$ because of a high PL background.

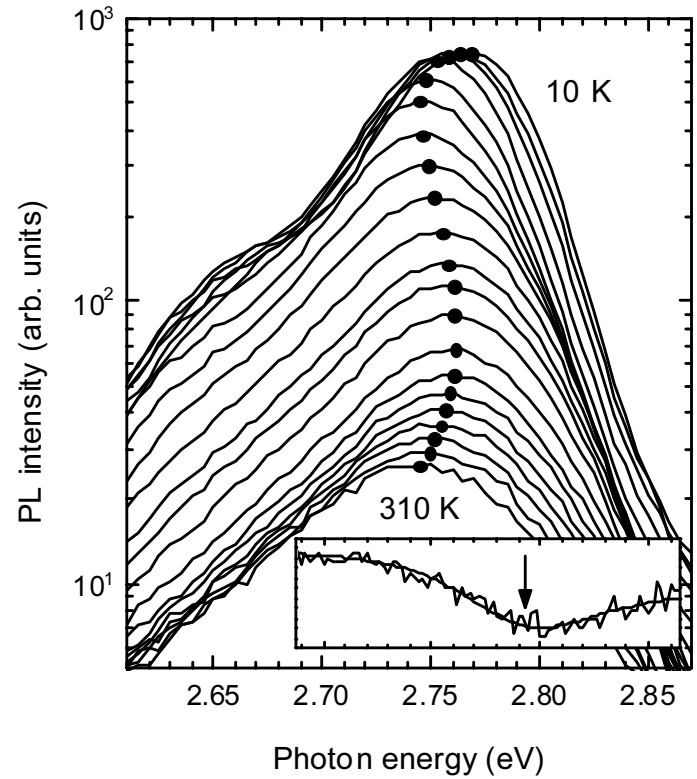

Fig. 1 Evolution of the PL spectra with temperature in InGaN/GaN MQWs. The temperature is incremented by $15 \mathrm{~K}$ starting from $10 \mathrm{~K}$ (the uppermost spectrum) and ending with $310 \mathrm{~K}$ (the lowermost spectrum). The band peak positions are indicated by dots. The inset shows a PR spectrum at $295 \mathrm{~K}$ with the arrow indicating the band resonance energy.

The measured W-shaped temperature dependence of the full width at half maximum (FWHM) of the PL band is shown by points in Fig. 2. This dependence was fitted with a simulated one obtained by using a 2D Monte Carlo procedure [6] with the Miller-Abrahams rate for phonon-assisted exciton tunneling between the initial and final states $i$ and $j$ with the energies $E_{i}$ and $E_{j}$, respectively,

$$
v_{i \rightarrow j}=v_{0} \exp \left(-\frac{2 r_{i j}}{\alpha}-\frac{E_{j}-E_{i}+\left|E_{j}-E_{i}\right|}{2 k_{B} T}\right) \text {. }
$$


Here $r_{i j}$ is the distance between the states, $\alpha$ is the decay length of the exciton wave function, and $v_{0}$ is the attempt-to-escape frequency. The simulation procedure involved a random set of localized states with the sheet density $N$. Gaussian dispersion of the localization energies was assumed with the peak at the mean exciton energy and the standard deviation of the band potential profile fluctuations $\sigma$. Each generated exciton terminated the hopping process by recombination with the probability $\tau_{0}^{-1}$ and the energy of the lethal localized state was scored to the emission spectrum $S_{0}(h v)$. To accomplish the quantitative fitting of the simulation results with the experimentally determined linewidth at low temperatures, an additional inhomogeneous broadening $\Gamma$ was introduced by transforming the initial emission spectrum $S_{0}(h v)$ to

$$
S(h v)=\int S_{0}\left(h v^{\prime}\right) G\left(\Gamma, h v-h v^{\prime}\right) d h v^{\prime},
$$

where $G(\Gamma, h v)$ is a Gaussian function with the standard deviation $\Gamma$.

Line in Fig. 2 represents the results of the fit for $N \alpha^{2}=1, v_{0} \tau_{0}=3 \times 10^{5}, \sigma=31 \mathrm{meV}$, and $\Gamma=29 \mathrm{meV}$. In the initial temperature range from $10 \mathrm{~K}$ to $150 \mathrm{~K}$, the variation of the linewidth depends on the products $N \alpha^{2}$ and $v_{0} \tau_{0}$, reflecting the spatial and temporal properties of the hopping process, respectively. A crossover from a nonthermalized to thermalized energy distribution of excitons is represented by a kink in the temperature dependence of the linewidth, with the characteristic temperature of about $150 \mathrm{~K}$ being determined by the standard deviation of the band potential, $\sigma$. The thermalized exciton energy distribution results in an almost constant linewidth $2 \sqrt{\left(\sigma^{2}+\Gamma^{2}\right) \ln 4}$ right above the crossover temperature (150$180 \mathrm{~K})$. The further increase of the linewidth above $180 \mathrm{~K}$ can be attributed to phonon broadening of the radiative transitions and to the influence of delocalized excitons that are not taken into account in our model.

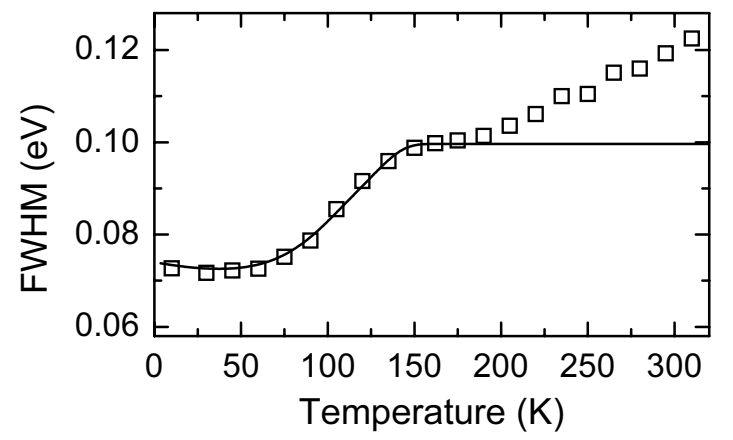

Fig. 2 Temperature dependence of the full width at half maximum of the PL band in 2.5-nm InGaN/GaN MQWs. Points depict experimental data; solid line shows the result of Monte Carlo simulation of exciton hopping for the roughness of the band potential profile $\sigma=31 \mathrm{meV}$ and inhomogeneous broadening $\Gamma=29 \mathrm{meV}$.

Note that the band potential roughness $\sigma$ accounts for dispersion of the localized states that the excitons are hopping through, meanwhile the inhomogeneous broadening $\Gamma$ accounts for dispersion in the mean exciton energy among the isolated regions that the excitons are hopping within. We attribute these regions to the In-rich regions, which have been revealed in InGaN by numerous observations, e.g. by high-resolution microscopy and X-ray microanalysis $[9,10]$. The inhomogeneous broadening is due to the fluctuations of the average indium content within the In-rich regions [7] and, in quantum wells, additionally due to the well-width fluctuations, which modulate the quantum confinement energy, and fluctuations of piezoelectric polarization, which result in the inhomogenous red shift due to the QCSE [11].

Solid dots in Fig. 3 depict the temperature dependence of the actual average exciton energy (with the QCSE included), which was reconstructed using the experimental data on the PL peak position (Fig. 1) and the temperature behavior of the Stokes shift deduced from the simulated spectra. The reconstructed dependence exhibited a Bose-Einstein-like behavior described as $E(T)=E(0)-\lambda[\exp (\theta / T)-1]^{-1}$ with the best-fit parameters $\lambda=0.154 \mathrm{eV}, \theta=379 \mathrm{~K}$, and $E(0)=2.845 \mathrm{eV}$ (line in Fig. 3). The obtained dependence is seen to agree with the PR data (open rectangles in Fig. 3). This suggests that Monte Carlo simula- 
tion of exciton hopping, although being a rather indirect method, can be used for the determination of the fundamental optical transition energy in InGaN MQWs, especially, when other techniques are difficult to apply (like in our case at low temperatures, where PR is difficult to measure because of the PL background).

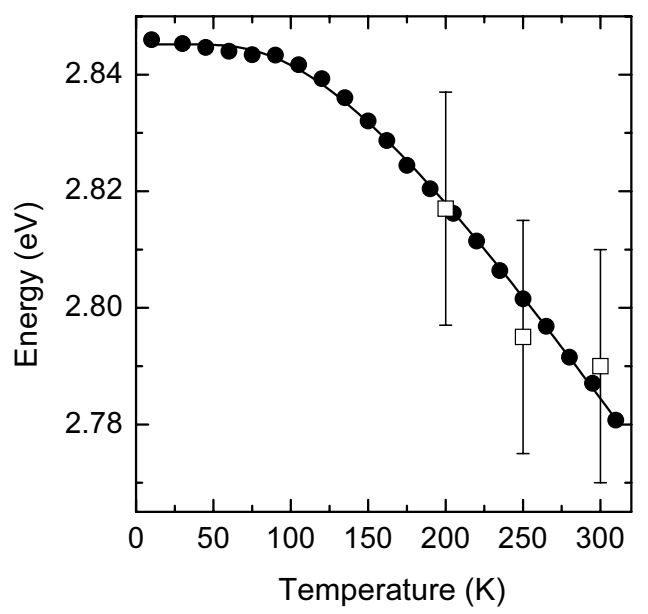

Fig. 3 Reconstructed average exciton energy (solid dots) as a function of temperature in InGaN/GaN MQWs. Line represents the best fit by using a Bose-Einstein-like formula. Open rectangles show the energy of the PR resonance.

4 Conclusion An analysis of temperature behavior of the PL linewidth in InGaN/GaN MQWs by using Monte Carlo simulation of exciton hopping was demonstrated. The simulation revealed the scale of potential fluctuations within individual In-rich regions $(31 \mathrm{meV})$ and inhomogeneous broadening $(29 \mathrm{meV})$ due to fluctuations in the average exciton energy among individual In-rich regions. The Stokes shift of the PL band deduced from the simulation and the measured PL peak positions were used to reconstruct the temperature dependence of the average exciton energy, which exhibited a characteristic Bose-Einstein-like behavior and was shown to be in a fair agreement with the PR data.

Acknowledgements This research was partially supported by the joint Lithuanian-Latvian-Taiwan grant. A. Ž. and G. T. acknowledge the Lithuanian Ministry of Education and Science for their Fellowship.

\section{References}

[1] S. Chichibu, T. Sota, K. Wada, and S. Nakamura, J. Vac. Sci. Technol. B 16, 2204 (1998).

[2] Y.-H. Cho, G. H. Gainer, J. B. Lam, J. J. Song, W. Yang, and W. Jhe, MRS Internet J. Nitride Semicond. Res. 5S1, W11.57 (2000).

[3] P. G. Eliseev, P. Perlin, J. Lee, and M. Osinski, Appl. Phys. Lett. 71, 569 (1997).

[4] Y.-H. Cho, T. J. Schmidt, S. Bidnyk, G. H. Gainer, J. J. Song, S. Keller, U. K. Mishra, and S. P. DenBaars, Phys. Rev. B 61, 7571 (2000).

[5] D. Monroe, Phys. Rev. Lett. 54, 146 (1985).

[6] S. D. Baranovskii, R. Eichmann, and P. Thomas, Phys. Rev. B 58, 13081 (1998).

[7] K. Kazlauskas, G. Tamulaitis, A. Žukauskas, M. A. Khan, J. W. Yang, J. Zhang, G. Simin, M. S. Shur, and R. Gaska, Appl. Phys. Lett. 83, 3722 (2003).

[8] C. Wetzel, T. Takeuchi, S. Yamaguchi, H. Katoh, H. Amano, and I. Akasaki, Appl. Phys. Lett. 73, 1994 (1998).

[9] Y. Narukawa, Y. Kawakami, M. Funato, S. Fujita, S. Fujita, and S. Nakamura, Appl. Phys. Lett. 70, 981 (1997).

[10] Y. S. Lin, K. J. Ma, C. Hsu, S. W. Feng, Y. C. Cheng, C. C. Liao, C. C. Yang, C. C. Chou, C. M. Lee, and J. I. Chyi, Appl. Phys. Lett. 77, 2988 (2000).

[11] A. Hangleiter, J. Lumin. 87-89, 130 (2000). 